\title{
Factores de corrección para predecir la resistencia a compresión in-situ de un hormigón autocompactante
}

\section{Correction factors to predict the in-place compressive strength of a self-compacting concrete}

\author{
L. Rojas-Henao ${ }^{(*)}$, J. Fernández-Gómez ${ }^{(* *)}$, J.C. López-Agüi(**)
}

Recepción/Received: 4-IX-2012

Aceptación/Accepted: 12-IX -2013

\section{RESUMEN}

Este estudio tiene como propósito conocer si algunos factores de corrección que se utilizan para predecir la resistencia a compresión in situ a través de probetas testigo son de directa aplicación en los hormigones autocompactantes (HAC). Dichos factores están relacionados con el diámetro, la dirección de extracción, la humedad y algunas variables intrínsecas del testigo, así como el nivel resistente del hormigón.

Además, para el HAC de este estudio se han obtenido los factores que convierten la resistencia de una probeta cúbica de $15 \times 15 \mathrm{~cm}$ en la de una cilíndrica de $30 \times 15 \mathrm{~cm}$.

Los resultados muestran que los factores de corrección propuestos por la EHE-08 y la ACI 214.4R-10 sobrevaloran la resistencia a compresión in situ del HAC analizado. Asimismo, se han encontrado diferencias en los factores que convierten la resistencia de la probeta cúbica en la de la cilíndrica según el Código Modelo (2010), pero similitudes según la EHE-08.

Palabras clave: hormigón autocompactante; resistencia a compresión in situ; probetas testigo; probetas cilíndricas; probetas cúbicas.

\section{ABSTRACT}

The present study aimed to determine whether certain correction factors used in the in-place prediction of compressive strength with concrete cores are directly applicable to self-consolidating concretes (SCCS). The parameters considered were core diameter, casting direction, core moisture, a number of variables intrinsic to cores, and concrete strength.

Factors were also established for converting SCC $15 \times 15-\mathrm{cm}$ cubic specimen strength into $30 \times 15-\mathrm{cm}$ cylindrical specimen strength.

The findings show that the correction factors recommended in EHE-08 and ACI 214.4R-10 overestimate the in-place compressive strength of the SCC analysed. The factors found for converting cubic into cylindrical specimen strength, in turn, were observed to differ from the values set out in the 2010 Model Code, but to be similar to the EHE-08 code proposals.

Keywords: self-consolidating concrete; in-place compressive strength; core samples; cylindrical specimens; cubic specimens.

\footnotetext{
(*) Federación Interamericana del Cemento (FICEM) (Colombia).

(**) Universidad Politécnica de Madrid (España).
} 


\section{INTRODUCCIÓN}

\subsection{Estimación de la resistencia in situ a través de probetas testigo}

El ensayo a compresión de probetas testigo es el método más directo para determinar la resistencia a compresión in situ de una estructura de hormigón (1). El análisis e interpretación de los resultados de la rotura de probetas testigo en Hormigones Vibrados Tradicionalmente (HVT) ha sido tema de discusión de numerosos estudios. Sin embargo, no existen estudios suficientes sobre su adecuación y correcta interpretación en algunos de los nuevos tipos de hormigones, específicamente en el HAC.

Con el objeto de predecir la resistencia a compresión in situ del hormigón a través de la resistencia a compresión de la probeta testigo, la norma americana ACI-214.4R-10 (1) considera el principio de superposición de efectos mediante factores de corrección. Dicha predicción la realiza mediante la expresión [1]:

$$
\tilde{f}_{c}=f_{c, \text { core }} F_{l / d} F_{\phi} F_{\text {moist }} F_{d} \text {, }
$$

donde $\tilde{f}_{c}$ es la resistencia equivalente in situ, $f_{c, \text { core }}$ es la resistencia del testigo, $F_{l / d}$ es el factor de corrección para la relación longitud/diámetro del testigo, $F_{\phi}$ es el factor de corrección para el diámetro del testigo, $F_{\text {moist }}$ es el factor de corrección para el contenido de humedad del testigo y $F_{d}$ es el factor de corrección por el daño del testigo durante la extracción. Los valores medios para estos factores se observan en la Tabla 1.

A este respecto la Instrucción Española de Hormigón Estructural EHE-08 (2) no define ningún procedimiento para la predicción de la resistencia de la probeta moldeada a través de la resistencia del testigo. La citada instrucción únicamente señala en sus comentarios que la

\section{INTRODUCTION}

\subsection{Estimate of in-place strength in core samples}

Core testing is the most direct method for determining the compressive strength of in-place concrete structures (1). The analysis and interpretation of core failure in traditionally vibrated concrete (CVC) have been the object of many a study. Nonetheless, neither the suitability of this procedure nor the interpretation of the results has been sufficiently studied for other types of concrete, selfconsolidating concrete (SCC) in particular.

For the intents and purposes of predicting in-place concrete compressive strength from core strength, American standard ACI-214.4R-10 (1) defines a series of correction factors, inter-related as shown below [1]:

Tabla 1 / Table 1

Factores de corrección para convertir la resistencia del testigo en resistencia equivalente in situ. Según ACI-214.4R-10. Correction factors for converting core strength into equivalent in-place strength as per ACI-214.4R-10.

\begin{tabular}{|l|c|c|}
\hline \multirow{2}{*}{ Factor } & Descripción / Description & Valor medio / Mean value * \\
\hline \multirow{3}{*}{$F_{I / d}$} & Saturados durante 48 horas / Soaked for 48 hours & $1-\left\{0.117-\alpha f_{C, \text { core }}\right\}(2-1 / d)^{2}$ \\
\cline { 2 - 3 } & Secos al aire / Air-dried & $1-\left\{0.144-\alpha f_{C, c o r e}\right\}(2-1 / d)^{2}$ \\
\cline { 2 - 3 } & Sin ninguna conservación / As received & $1-\left\{0.130-\alpha f_{C, \text { core }}\right\}(2-1 / d)^{2}$ \\
\cline { 2 - 3 }$F_{\phi}$ & $50 \mathrm{~mm}$ & 1.06 \\
\hline \multirow{2}{*}{$F_{\text {moist }}$} & $100 \mathrm{~mm}$ & 1.00 \\
\cline { 2 - 3 } & $150 \mathrm{~mm}$ & 0.98 \\
\cline { 2 - 3 }$F_{d}$ & Saturados durante 48 horas / Soaked for 48 hours & 1.09 \\
\cline { 2 - 3 } & Secos al aire / Air-dried & 0.96 \\
\hline
\end{tabular}

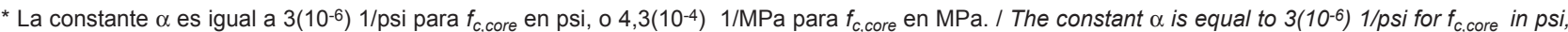
or $4.3\left(10^{-4}\right) \quad 1 / / \mathrm{MPa}$ for $f_{c, \text { core }}$ in $\mathrm{MPa}$. 
resistencia del testigo se ve afectada por el proceso de extracción, en el cual se genera un cierto nivel de daño en el propio hormigón del testigo mediante la aparición de microfisuras que posiblemente puedan afectar los resultados obtenidos. Dicha instrucción alude que, a falta de datos especificados para cada caso, es habitual considerar que para hormigones normales el efecto descrito provoca una disminución de aproximadamente un $10 \%$ en la resistencia.

El presente estudio ha adoptado el modelo multiplicativo de ACI-214.4R-10, considerado como variables de estudio el diámetro, la dirección de extracción y la humedad de las probetas testigo, además del nivel resistente del hormigón y un conjunto de variables intrínsecas de las probetas que son difíciles de medir por sí solas (posible microfisuración y debilitamiento de los áridos durante el proceso de corte y extracción, el efecto pared, las condiciones de contorno, etc.). Mayor información sobre estas variables intrínsecas se puede consultar en Pérez (3).

Para la selección de la variable predicha, es decir la resistencia a compresión in situ, se han considerado condiciones de hormigonado lo más parecidas posible a las presentadas en los elementos in situ (Bloques de HAC). Para ello se fabricaron probetas cilíndricas moldeadas de $15 \times 30 \mathrm{~cm}$ con la misma amasada de los bloques de HAC y con condiciones similares, es decir, ninguna compactación mecánica y con un curado en condiciones de obra. En este estudio la resistencia a compresión obtenida de estas probetas cilíndricas es denominada "resistencia equivalente in situ" $f_{c y l \text {, inplace. }}$

Asimismo para una mejor correlación en la predicción de la resistencia in situ, se ha elegido a la probeta moldeada cilíndrica y no a la cúbica debido a la similitud en la forma y esbeltez con las probetas testigo.

\subsection{Relación de resistencias entre probetas cilíndricas y probetas cúbicas}

En relación con las probetas moldeadas, los tipos de probetas más comúnmente usadas para determinar la resistencia a compresión del hormigón son las cúbicas y las cilíndricas. Las probetas cúbicas de $15 \times 15 \mathrm{~cm}$ se utilizan en países como Gran Bretaña, Alemania y en algunas otras partes de Europa, mientras que en otros países como Estados Unidos, Corea del Sur, Francia, Canadá y Australia la probeta normalizada es la cilíndrica de $15 \times 30 \mathrm{~cm}$ (4). En España se permite el uso de los dos tipos de probetas, así lo indica la vigente Instrucción Española de Hormigón Estructural EHE-08.

En el caso de España es de gran importancia emplear factores de corrección si se ensayan probetas cúbicas. of damage to the concrete, thereby impacting the test findings. It adds that, in the absence of data for a given specific case, that effect is normally assumed to reduce strength by approximately $10 \%$.

The present study adopted the multiplicative approach described in ACI-214.4R-10, in which the variables studied were core diameter, casting direction and core moisture condition, along with concrete strength and certain intrinsic properties that are difficult to measure directly (possible microcracking during drilling, pop-out of damaged coarse aggregate particles during testing, wall or boundary effects and so on). Further information on these intrinsic variables can be found in Pérez (3).

To find the response variable, i.e., in-place compressive strength, the specimens were cast under conditions as close as possible to the conditions used for in-place elements (SCC blocks). To that end, $15 \times 30-\mathrm{cm}$ cylindrical specimens were cast from the same batch of concrete as the SCC blocks and under similar conditions: i.e., with no mechanical consolidation and cured in worksite conditions. In this study the compressive strength found for these cylindrical specimens is called "equivalent in-place strength" and symbolised as $f_{c y l, \text { inplace. }}$

Given their greater similarity in shape and slenderness to cores, cylindrical were chosen over cubic specimens to obtain a higher correlation with the in-place strength values.

\subsection{Relationship between cylindrical and cubic specimen strength}

Cubic and cylindrical specimens are the two types of samples most commonly used to determine concrete compressive strength. Fifteen-centimetre cubic specimens are used in some European countries, including the United Kingdom and Germany, while in the United States, South Korea, France, Canada and Australia the standard specimen is a $15 \times 30-\mathrm{cm}$ cylinder (4). The Spanish structural concrete code, Code EHE-08, makes provision for both types of specimens.

The use of correction factors is extremely important when cubic specimens are tested to determine member 
Cada vez más los laboratorios eligen para sus ensayos este tipo de probeta que a diferencia de las cilíndricas no precisan refrentado ni pulido lo cual contribuye al equilibrio ecológico y a la protección del medio ambiente.

Para el caso del HAC pocos investigadores han estudiado la transformación de los resultados de resistencia obtenidos en probeta cúbica en función de la probeta cilíndrica. Al respecto, Domone (5), en un amplio estudio recopila los resultados de 28 investigaciones, donde clasifica la resistencia a compresión (en probeta cúbica o cilíndrica) en función de la relación agua/cemento y del tipo de árido. Además encuentra que la ratio entre la resistencia de la probeta cilíndrica y la probeta cúbica $\left(f_{c, c y l} / f_{c, c u b}\right)$ varía alrededor de 0.8 para resistencias de $30 \mathrm{MPa}$ y cerca de 1.00 para resistencias de $90 \mathrm{MPa}$.

\section{PROGRAMA EXPERIMENTAL}

El HAC de este estudio fue proporcionado por una empresa de prefabricados Española, y forma parte de su producción habitual (ver las proporciones de la mezcla en la Tabla 2). El cemento utilizado ha sido el Portland tipo I52.5R y el tamaño máximo del árido de $12 \mathrm{~mm}$. El rango de resistencias de las mezclas obtenido fue de 20 MPa a $80 \mathrm{MPa}$. Por otro lado los resultados del ensayo de escurrimiento variaron entre $665 \mathrm{~mm}$ y $750 \mathrm{~mm}$ y los del coeficiente de bloqueo obtenidos del ensayo de la caja en $L$ variaron entre 0,83 y 0,95 .

Con la mezcla de HAC se fabricaron 30 bloques de $50 \times 50 \times 100 \mathrm{~cm}$. A la edad de 22 días se extrajeron 12 testigos por cada bloque, seis en sentido perpendicular (horizontal) y los otros seis en sentido paralelo (vertical) a la dirección de hormigonado. Los diámetros considerados fueron de $5 \mathrm{~cm}, 7,5 \mathrm{~cm}$ y $10 \mathrm{~cm}$, con una esbeltez igual a 2 . El número total de testigos extraídos fue de 360 la mitad de ellos fueron sumergidos en agua durante 48 horas y los restantes estuvieron en condiciones secas en ambiente de laboratorio. La planeidad y paralelismo de las dos caras de los testigos se consiguió por medio del refrentado de mortero de azufre.

Adicionalmente se fabricaron tres probetas cilíndricas de $15 \times 30 \mathrm{~cm}$ y dos probetas cúbicas de $15 \times 15 \mathrm{~cm}$ por strength. In Spain, laboratories tend to choose this more environmentally friendly type of specimen, for unlike cylindrical samples, they do not need to be capped or polished.

Very few researchers have studied the conversion of cubic to cylindrical specimen strength values in SCC. In an extensive review, Domone (5) classified the cubic or cylindrical compressive strength values reported in 28 papers in terms of the water/cement ratio and type of aggregate. That author found that the cylindrical / cubic specimen strength ratio $\left(f_{c, c y l} / f_{c, c u b}\right)$ ranged from around 0.8 for strengths of $30 \mathrm{MPa}$ to around 1.00 for strengths of $90 \mathrm{MPa}$.

\section{EXPERIMENTAL}

The SCC for this study consisted of the standard material manufactured by a Spanish precasting company (batching details in Table 2) using I52.5R portland cement and coarse aggregate with a top size of $12 \mathrm{~mm}$. The characteristics of the concrete mixtures were: strength from 20 to $80 \mathrm{MPa}$; slump flow test of 665 to $750 \mathrm{~mm}$; and L-box blocking ratios of 0.83 to 0.95 .

The SCC mixes were used to cast thirty $50 \times 50 \times 100-\mathrm{cm}$ blocks. Twenty-two days after casting, twelve samples were cored from each block, six perpendicular (horizontal) and six parallel (vertical) to the direction of casting. The cores were $5,7.5$ or $10 \mathrm{~cm}$ in diameter and had a slenderness ratio of 2. Half of the total 360 cores drilled were soaked in water for 48 hours and the rest were allowed to dry under laboratory conditions. Both ends of the cores were capped with sulfur mortar to ensure that they would be both perfectly flat and parallel to one another.

In addition, three $15 \times 30-\mathrm{cm}$ cylindrical and two $15-\mathrm{cm}$ cubic pores were prepared per block, for a total of 150

Tabla 2 / Table 2

Proporciones de la mezcla de HAC*.

SCC batching proportions*.

\begin{tabular}{|c|c|c|c|c|c|c|c|}
\hline & $\begin{array}{l}\text { Árido fino / Fines } \\
\left(\mathrm{kg} / \mathrm{m}^{3}\right)\end{array}$ & $\begin{array}{c}\text { Árido grueso / } \\
\text { Coarse aggregate } \\
\left(\mathrm{kg} / \mathrm{m}^{3}\right)\end{array}$ & $\begin{array}{l}\text { Cemento / Cement } \\
\left(\mathrm{kg} / \mathrm{m}^{3}\right)\end{array}$ & $\begin{array}{l}\text { Aditivo / } \\
\text { Admixture } \\
\left(\mathrm{L} / \mathrm{m}^{3}\right)\end{array}$ & $\begin{array}{c}\text { Filler } \\
\left(\mathbf{k g} / \mathrm{m}^{3}\right)\end{array}$ & $\begin{array}{c}\text { Agua / Water } \\
\qquad\left(\mathrm{L} / \mathrm{m}^{3}\right)\end{array}$ & $\begin{array}{c}\text { Relación } \\
\text { A/C I } \\
\text { W/c ratio }\end{array}$ \\
\hline $20-40 \mathrm{MPa}$ & 943 & 720 & 330 & 5.5 & 200 & 150 & 0.45 \\
\hline $50-80 \mathrm{MPa}$ & 800 & 840 & 420 & 6.5 & 150 & 190 & 0.45 \\
\hline
\end{tabular}

* Se indican los valores medios de las proporciones. $/$ * The values shown are means. 
cada bloque, es decir, un total de 150 probetas moldeadas. El procedimiento empleado para el refrentado de las probetas cilíndricas fue mediante mortero de azufre.

Finalmente todas las probetas moldeadas y los testigos fueron ensayados a compresión a la edad de 28 días, según la norma UNE-EN 12390-3 (6).

Para simular el hormigonado con altas cuantías de armadura, 14 de los 30 bloques fueron fabricados vertiendo el HAC a través de una malla de barras de $12 \mathrm{~mm}$ de diámetro espaciadas cada $20 \mathrm{~mm}$ y situada sobre el borde superior del encofrado (Figura1). Las condiciones de curado de los bloques y probetas moldeadas fueron las mismas, teniendo en cuenta condiciones similares a las que se presentan habitualmente en la práctica.

Los bloques de HAC también fueron utilizados para otros estudios relacionados con ensayos no destructivos en el hormigón endurecido $(7,8)$.

La información descrita en este estudio relacionada con el cociente entre la resistencia de la probeta cilíndrica moldeada y la resistencia del testigo en los HVT ha sido tomada de la base de datos de la tesis doctoral de Rojas, L. (7). Esta base de datos contiene 44 resultados obtenidos de otras investigaciones que presentaron especificaciones similares en las probetas ensayadas.

\section{RESULTADOS Y ANÁLISIS}

En la Tabla 3 se muestran los resultados de la resistencia a compresión de las probetas testigo clasificadas según la condición de humedad, la dirección de extracción y el diámetro del testigo. Asimismo en la Tabla 4 se muestran los resultados las resistencias a compresión de las probetas modeladas (cilíndricas y cúbicas) que fueron obtenidas de la misma amasada de cada elemento o bloque de HAC.

\subsection{Factores de corrección para la resistencia de probetas testigo en el pronóstico de la resistencia in situ}

Con el objeto de pronosticar la resistencia equivalente in situ a través de probetas testigo se ha desarrollado un procedimiento para la deducción de los factores de corrección que valora la influencia de las condiciones específicas de este estudio.

Este estudio ha tomado como referencia el modelo multiplicativo de ACI-214.4R-10. Con las siguientes consideraciones: cast specimens. The cylindrical specimens were also capped with sulfur mortar.

All the 28-day materials, cylindrical specimens and cores both, were compression-tested as specified in Spanish and European standard UNE-EN 12390-3 (6).

To simulate concrete with high steel ratios, in 14 of the 30 blocks the SCC was poured onto a lattice at the uppermost part of the forms, consisting of 12-mm diameter bars spaced at $20 \mathrm{~mm}$ (Figure 1). The blocks and cast specimens were cured under identical conditions that were similar to standard worksite specifications.

The SCC blocks were also used for other related hardened concrete studies involving non-destructive tests $(7,8)$.

The information on the ratio between cylindrical specimen and core sample strength in CVC used in this study was drawn from the author's PhD. thesis (Rojas (7)), a database containing 44 records reported by other authors using specimens similar to the ones tested.

\section{RESULTS AND ANALYSIS}

Table 3 gives the compressive strength values for the cores, classified by moisture condition, casting direction and core diameter. Table 4, in turn, lists the compressive strength findings for the (cylindrical and cubic) specimens cast with the same concrete as each SCC block.

\subsection{Correction factors for predicting in-place strength from core specimen strength}

A procedure was deduced to find the correction factors for the specific conditions prevailing in this study, with a view to predicting the equivalent in-place strength from the cores.

The reference used was the multiplicative approach described in standard ACI-214.4R-10 and the criteria adopted were as set out below. 
Tabla 3 / Table 3

Resultados de resistencias a compresión de las probetas testigo. Core compressive strength.

\begin{tabular}{|c|c|c|c|c|c|c|c|c|c|c|c|c|}
\hline \multirow{4}{*}{$\begin{array}{l}\text { Bloque HAC I } \\
\text { SCC block }\end{array}$} & \multicolumn{12}{|c|}{$f_{c, \text { core }}(\mathrm{MPa})$} \\
\hline & \multicolumn{6}{|c|}{ Secos / Air-dried } & \multicolumn{6}{|c|}{ Saturados / Wet } \\
\hline & \multicolumn{3}{|c|}{ Horizontal } & \multicolumn{3}{|c|}{ Vertical } & \multicolumn{3}{|c|}{ Horizontal } & \multicolumn{3}{|c|}{ Vertical } \\
\hline & $5 \mathrm{~cm}$ & $7.5 \mathrm{~cm}$ & $10 \mathrm{~cm}$ & $5 \mathrm{~cm}$ & $7.5 \mathrm{~cm}$ & $10 \mathrm{~cm}$ & $5 \mathrm{~cm}$ & $7.5 \mathrm{~cm}$ & $10 \mathrm{~cm}$ & $5 \mathrm{~cm}$ & $7.5 \mathrm{~cm}$ & $10 \mathrm{~cm}$ \\
\hline $1 \mathrm{SB}$ & 47.4 & 40.6 & 42.8 & 47.1 & 35.1 & 43.5 & 25.6 & 33.6 & 31.7 & 36.3 & 38.1 & 36.9 \\
\hline $2 S B$ & 68.7 & 65.4 & 63.2 & 59.2 & 67.2 & 66.7 & 40.9 & 61.2 & 61.7 & 33.1 & 56.6 & 57.4 \\
\hline $3 S B$ & 55.9 & 50.2 & 51.8 & 52.1 & 46.2 & 50.2 & 41 & 44.8 & 43.6 & 42 & 38.5 & 35.5 \\
\hline 4SB & 70.7 & 66.9 & 62.9 & 50.7 & 51.8 & 69 & - & 43.6 & 57.8 & 44.6 & 55.2 & 63.3 \\
\hline $5 S B$ & 31 & 25.7 & 24.2 & 32.6 & 25.4 & 24.2 & 18.2 & 18.6 & 20.7 & 29.1 & 22.1 & 20.1 \\
\hline $6 S B$ & 63.1 & 61.7 & 65.1 & 62.4 & 59.5 & 58.3 & 52.9 & 53.4 & 54.7 & 50.3 & 53.6 & 52.4 \\
\hline $7 S B$ & 26.3 & 23 & 25 & 24.4 & 22.2 & 24 & 22.4 & 18.5 & 22.7 & 18.6 & 18.5 & 20.1 \\
\hline $8 S B$ & 56.3 & 44.1 & 49.6 & 54.1 & 54.7 & 54.7 & 41.8 & 43.8 & 47.6 & 50.8 & 40.5 & 48.7 \\
\hline $9 S B$ & 42.9 & 48.6 & 39.2 & 44.7 & 47.5 & 45.1 & 41.5 & 33.7 & 42.2 & 41.7 & 39.5 & 35.5 \\
\hline $10 \mathrm{SB}$ & 43.5 & 44.1 & 47.9 & 45.5 & 44 & 40.6 & 35.2 & 33.9 & 41.7 & 35 & 42.1 & 39.5 \\
\hline 11SB & 54.2 & 51.6 & 65.3 & 60.2 & 64 & 66.3 & 56.1 & 48.6 & 57 & 60.4 & 40.6 & 60 \\
\hline $12 S B$ & 65.8 & 62.8 & 65.5 & 70.5 & 65.2 & 73.1 & 49.8 & 59.7 & 59.3 & 63.5 & 61.9 & 68.7 \\
\hline $13 S B$ & 56.1 & 56.9 & 61.7 & 51.3 & 58.6 & 57.3 & 46.2 & 53.7 & 55.1 & 49 & 51.5 & 54 \\
\hline 14SB & 65.3 & 65.6 & 64.2 & 63.1 & 67.5 & 67.4 & 51.4 & 51.3 & 56.7 & 59.1 & 57.5 & 51.1 \\
\hline $15 S B$ & 46.8 & 47.2 & 51.4 & 53 & 51.9 & 49.1 & 35 & 45.1 & 47.3 & 44.5 & 43.5 & 46.3 \\
\hline $16 S B$ & 46.5 & 65.1 & 64.6 & 53.8 & 61.5 & 57.7 & 57.6 & 54.6 & 60.5 & 51.6 & 42.4 & 60.8 \\
\hline $1 \mathrm{CB}$ & 48.1 & 44.7 & 45.7 & 55.8 & 46 & 56.2 & 40.4 & 40.8 & 51.9 & 37.6 & 44.3 & 49.1 \\
\hline $2 \mathrm{CB}$ & 64.8 & 58.5 & 62.5 & 59.7 & 60.5 & 61.6 & 43.7 & 43.5 & 53.1 & 59.7 & 55.9 & 55.5 \\
\hline $3 \mathrm{CB}$ & 51.9 & 54.5 & 59.1 & 50.7 & 49.3 & 55.4 & 46.5 & 50.3 & 41.7 & 49.3 & 48.2 & 37.9 \\
\hline $4 \mathrm{CB}$ & 72.7 & 62.7 & 63.9 & 72.4 & 52.6 & 68.1 & 56.4 & 60.1 & 50.7 & 49.3 & 46.8 & 54.9 \\
\hline $5 \mathrm{CB}$ & 59.6 & 53.8 & 56.5 & 52.4 & 57.3 & 50.4 & 48.5 & 41.3 & 40.2 & 42.6 & 49.6 & 48.3 \\
\hline $6 \mathrm{CB}$ & 53.8 & 52.3 & 59.1 & 44.9 & 41.2 & 51 & 44.2 & 43.8 & 38.9 & 48.2 & 45.2 & 49.2 \\
\hline $7 \mathrm{CB}$ & 48.3 & 42.4 & 44.3 & 45 & 40.1 & 43.6 & 40.2 & 35.9 & 38.9 & 32.5 & 32.5 & 42.8 \\
\hline $8 \mathrm{CB}$ & 64.8 & 69.1 & 60.5 & 66.4 & 66 & 60.1 & 52.4 & 45.1 & 57.8 & 58.4 & 53.7 & 53 \\
\hline $9 \mathrm{CB}$ & 55.8 & 56 & 44 & 50.6 & 49.7 & 54.3 & 49.7 & 49.3 & 39.7 & 46.4 & 43 & 41.8 \\
\hline $10 \mathrm{CB}$ & 62.6 & 57.5 & 58.7 & 63 & 45 & 61.4 & 42.9 & 55.9 & 57.3 & 55.6 & 51 & 48.6 \\
\hline $11 \mathrm{CB}$ & 50.6 & 54.3 & 55.5 & 43.3 & 55.9 & 55.5 & 42.8 & 42.3 & 48.9 & 41.3 & 40.3 & 50.1 \\
\hline $12 \mathrm{CB}$ & 59.9 & 68.8 & - & 46.7 & 71.2 & 74.8 & 49.5 & 41.9 & 63 & 53.3 & 58.1 & 59.5 \\
\hline $13 \mathrm{CB}$ & 56.1 & 51.3 & 57.2 & 51.6 & 48.3 & 53.6 & 48.3 & 46.1 & 47.5 & 37.2 & 41.9 & 47.3 \\
\hline $14 \mathrm{CB}$ & 62.2 & 60.3 & 55.8 & 56.2 & 55 & 56 & 48.1 & 48.1 & 48.4 & 50 & 51.2 & 53.4 \\
\hline
\end{tabular}

Se ha considerado como la resistencia más próxima a la resistencia a compresión in situ la obtenida en probeta cilíndrica moldeada de $15 \times 30 \mathrm{~cm}$, debido a que se ha fabricado y conservado bajo condiciones lo más parecidas posibles a la de los bloques de HAC. Por lo tanto se utilizará la notación [2]
The strength found for the $15 \times 30-\mathrm{cm}$ cylindrical specimens was regarded as closest to the in-place compressive strength, inasmuch as the SCC specimens and their respective blocks were manufactured and stored under conditions as similar as possible. The assumption was, then [2]:

$$
f_{c, c y l}=f_{c y l, \text { inplace }}
$$

donde $f_{c, c y l}$ es la resistencia a compresión de la probeta cilíndrica y $f_{c y l \text {,inplace }}$ es la resistencia equivalente in situ.

Se ha elegido a la probeta moldeada cilíndrica y no a la cúbica debido a la similitud en la forma y esbeltez con las probetas testigo. where $f_{c, c y l}$ is the compressive strength of the cylindrical specimen and $f_{c y l \text {,inplace }}$ the equivalent in-place strength.

Cylindrical were chosen over cubic specimens because of their similarity to the cores, in form and slenderness ratio. 
Tabla 4 / Table 4

Resultados de resistencias a compresión de las probetas moldeadas. Specimen compressive strength values.

\begin{tabular}{|c|c|c|c|c|c|}
\hline \multirow{2}{*}{$\begin{array}{c}\text { Bloque HAC I } \\
\text { SCC block }\end{array}$} & \multicolumn{3}{|c|}{$f_{C, C y /}(\mathrm{MPa})$ cilíndrica / cylindrical } & \multicolumn{2}{|c|}{$f_{c, c u b}(\mathrm{MPa})$ cúbica / cubic } \\
\hline & 37.8 & 39.9 & 40.4 & 41.2 & 40.7 \\
\hline $2 S B$ & 64.9 & 63.5 & 63.3 & 71.6 & 69.8 \\
\hline $3 S B$ & 51.2 & 49.9 & 51 & 57.1 & 55 \\
\hline $4 S B$ & 67.8 & 68.4 & 68.5 & 78.3 & 77.5 \\
\hline $5 S B$ & 25.7 & 26.7 & 25.4 & 28.6 & 28.6 \\
\hline $6 S B$ & 81.4 & 80.6 & 77.6 & 81.7 & 79.9 \\
\hline $7 \mathrm{SB}$ & 21.4 & 19.8 & 21.9 & 30 & 29.6 \\
\hline $8 S B$ & 55.5 & 56.7 & 57.3 & 60 & 59.7 \\
\hline 9SB & 44.8 & 45.8 & 42.4 & 46.1 & 47.4 \\
\hline $10 \mathrm{SB}$ & 47.2 & 47 & 43.8 & 48.9 & 49.4 \\
\hline 11SB & 66.8 & 68.2 & 64.7 & 73 & 73.5 \\
\hline $12 S B$ & 75.9 & 72.4 & 67.8 & 75.6 & 81.3 \\
\hline $13 \mathrm{SB}$ & 60.7 & 62.4 & 61.2 & 65.9 & 64.1 \\
\hline $14 S B$ & 72.6 & 74.8 & 70.7 & 74.7 & 72.5 \\
\hline $15 S B$ & 52 & 53 & 54 & 57.2 & 55.7 \\
\hline 16SB & 67.6 & 63.5 & 72.8 & 71.7 & 76.1 \\
\hline $1 \mathrm{CB}$ & 58.1 & 57.9 & 58.7 & 61.2 & 58.7 \\
\hline $2 \mathrm{CB}$ & 76.2 & 74.1 & 73.5 & 76.8 & 77.4 \\
\hline $3 \mathrm{CB}$ & 52.6 & 55.3 & 52.8 & 60.4 & 61.2 \\
\hline $4 \mathrm{CB}$ & 72.2 & 70.9 & 72 & 76.7 & 78.7 \\
\hline $5 \mathrm{CB}$ & 58.9 & 55.5 & 58 & 65.3 & 63.6 \\
\hline $6 \mathrm{CB}$ & 54.2 & 49.3 & 50 & 65.3 & 64.6 \\
\hline $7 \mathrm{CB}$ & 55 & 48 & 49.9 & 53.1 & 54.1 \\
\hline $8 \mathrm{CB}$ & 70 & 70.9 & 68.8 & 74.3 & 75.9 \\
\hline $9 \mathrm{CB}$ & 55.7 & 53 & 56.7 & 60.2 & 59.9 \\
\hline $10 \mathrm{CB}$ & 62.4 & 63.1 & 65.6 & 74.1 & 74.2 \\
\hline $11 \mathrm{CB}$ & 55.8 & 58.4 & 59.9 & 58 & 63 \\
\hline $12 \mathrm{CB}$ & 71.2 & 74.7 & 75 & 76.2 & 77.2 \\
\hline $13 \mathrm{CB}$ & 51.9 & 54.6 & 54.1 & 57.3 & 57.7 \\
\hline 14CB & 68.7 & 63.4 & 70.9 & 70.7 & 73.2 \\
\hline
\end{tabular}

En la búsqueda del modelo apropiado se ha conseguido estabilizar la varianza de los residuos mediante un modelo tipo cociente (7) con la siguiente expresión [3]:
The variance of residuals was stabilised with a quotient model (7) conforming to the following Equation [3]:

$$
\frac{\tilde{f}_{c, \text { core }}}{f_{c y l, \text { inplace }}}=b_{1}+b_{2} f_{c y l, \text { inplace }}+b_{3} \phi^{2}+b_{4} \text { MOIST }+b_{5} \text { DIR }+b_{6} f_{c y l, \text { inplace }} \cdot \phi^{2}+b_{7} f_{c y l, \text { inplace }} \cdot \text { MOIST }+b_{8} \phi^{2} \cdot \text { MOIST } \text {. }
$$

donde

$\tilde{f}_{c, \text { core }} / f_{\text {cyl,inplace }}$ Variable dependiente: cociente entre la resistencia a compresión de la probeta testigo y la resistencia equivalente in situ.

$\phi^{2}$, MOIST y DIR: Variables independientes: diámetro, condiciones de humedad y dirección de extracción del testigo.

Los resultados del análisis de regresión múltiple de la [3] revelaron un coeficiente de determinación de $\mathrm{R}^{2}=0,788$ lo cual indica una buena eficacia predictiva del modelo. where

$\tilde{f}_{c, \text { core }} / f_{\text {cyl,inplace }}: \quad$ dependent variable: ratio between core compressive strength and the equivalent in-place strength

$\phi^{2}$, MOIST and DIR: independent variables: diameter, moisture condition and casting direction

The determination coefficient resulting from the multiple regression performed with [3], $\mathrm{R}^{2}=0.788$, was an indication of the high predictive power of the model. 


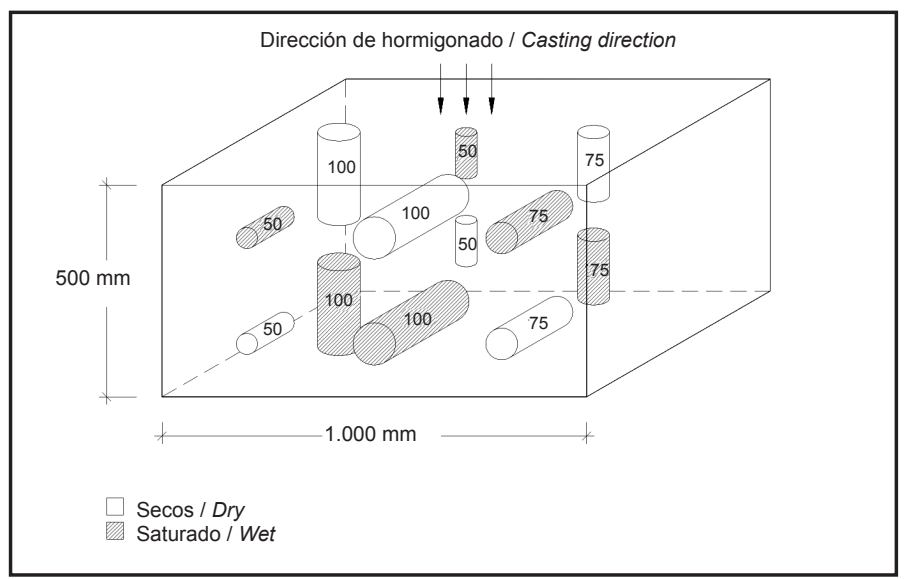

Figura 1. Esquema de la extracción de los testigos no está nítida.

Figure 1. Core drilling in SCC block.

En la Tabla 5 se muestran los resultados de la tabla ANOVA del modelo de la [3]. En el contraste de significación global, el estadístico $F=183,7$ supera ampliamente al cuantil crítico de la distribución $F$ para $\alpha=0,05$ y grados de libertad 6 y 1067, es decir, $F_{5}, 1068 ; 0.05=2,11$. Por lo tanto se acepta que el modelo de la [3] es estadísticamente significativo para explicar las variaciones de $f_{c, \text { core }} / f_{c y l, \text { inplace }}$.

Por otro lado en el modelo de la [3] el $p$-valor de todas las variables explicativas fue significativo, a excepción de la variable DIR (dirección de extracción del testigo) que presentó un $p$-valor del $66,9 \%$, muy superior al $5 \%$ adoptado.

Así pues, el modelo finalmente considerado con sus parámetros estimados y con la eliminación de la variable $D I R$ por su nulo grado de significación es el siguiente [4]:
Table 5 gives the ANOVA results for the model represented by [3]. The statistical-significance contrasts yielded an $F$ value (183.7) comfortably higher than the value for the critical quantile of the F-distribution for $\alpha=0.05$ and 6 and 1067 degrees of freedom, i.e. $\mathrm{F}_{5,1068 ; 0.05}=2,11$. The [3] model was consequently found to provide a statistically valid explanation of the variation in $f_{c, \text { core }} / f_{c y l \text {,inplace }}$.

Moreover, in the model represented by [3], the p-value was significant for all the explanatory variables except DIR (casting direction), whose p-value, at $66.9 \%$, was substantially higher than the 5\% cut-off.

Hence, the model and model parameters finally adopted, from which the DIR variable was excluded in light of its nil significance, was as shown below [4]:

$$
\begin{gathered}
\frac{\tilde{f}_{c, \text { core }}}{f_{c y l, \text { inplace }}}=1.329-0.006787 f_{\text {cyl,inplace }}-0.001759 \phi^{2}-0.2630 \text { MOIST }+ \\
+3.169 \times 10^{-5} f_{c y l, \text { inplace }} \cdot \phi^{2}+0.001521 f_{c y l, \text { inplace }} . \text { MOIST }+5.874 \times 10^{-4} \phi^{2} . \text { MOIST } .
\end{gathered}
$$

Tabla 5 / Table 5

Tabla ANOVA Modelo Ec. [3]. ANOVA table for the Equation [3] model.

\begin{tabular}{|c|c|c|c|c|c|}
\hline $\begin{array}{c}\text { Fuente de variación / } \\
\text { Source of variation }\end{array}$ & $\begin{array}{c}\text { Suma de cuadrados / } \\
\text { Sum of squares }\end{array}$ & $\begin{array}{c}\text { Grados de libertad / } \\
\text { Degrees of freedom }\end{array}$ & $\begin{array}{c}\text { Cuadrados medios / } \\
\text { Mean squares }\end{array}$ & $\begin{array}{c}\text { Estad. I } \\
\text { Statistic } \boldsymbol{F}\end{array}$ & $\begin{array}{c}\mathbf{p} \text {-valor / } \\
\boldsymbol{p} \text {-value }\end{array}$ \\
\hline Modelo / Mode/ & 9.082 & 6 & 1.514 & 183.7 & 0.000 \\
\hline Residual / Residual & 8.794 & 1067 & 0.008242 & & \\
\hline Total & 17.88 & 1073 & & & \\
\hline
\end{tabular}


A partir de este modelo de la [4] se pueden pronosticar los valores de $f_{c, \text { core }} / f_{c y l \text {,inplace }}$ para todos los conjuntos considerados en función del nivel resistente, diámetro y condición de humedad. En la Figura 2 se representan las seis rectas obtenidas de este modelo, con las siguientes ecuaciones [5-10]:
Equation [4] was consequently valid for predicting the $f_{c, \text { core }} / f_{c y l \text {, inplace values for all the sub-samples studied }}$ from the data on strength, diameter and moisture condition. Figure 2 shows the six regression lines obtained with the model, for the following Equations [5-10]:

Diámetro $10 \mathrm{~cm}$ en condición seca / Dry 10- cm diameter.

$$
\frac{f_{c, \text { core }(10, \mathrm{dry})}}{f_{c y l, \text { inplace }}}=1.153-0.003618 f_{\text {cyl, inplace }}
$$

Diámetro $7.5 \mathrm{~cm}$ en condición seca / Dry 7.5- cm diameter.

$$
\frac{f_{c, \text { core }}(7,5, \mathrm{dry})}{f_{c y l, \text { inplace }}}=1.230-0.005004 f_{c y l \text {, inplace }}
$$

Diámetro $5 \mathrm{~cm}$ en condición seca / Dry 5- cm diameter.

$$
\frac{f_{c, \text { core }(5, \mathrm{dry})}}{f_{c y l, \text { inplace }}}=1.285-0.005994 f_{c y l \text {, inplace }}
$$

Diámetro $10 \mathrm{~cm}$ en condición saturada / Wet 10- cm diameter:

$$
\frac{f_{c, \text { core }(10, \text { wet })}}{f_{c y l, \text { inplace }}}=0.9484-0.002097 f_{c y l \text {, inplace }}
$$

Diámetro $7.5 \mathrm{~cm}$ en condición saturada / Wet 7.5- cm diameter:

$$
\frac{f_{c, \text { core }(7,5, \text { wet })}}{f_{c y l \text {, inplace }}}=0.997-0.003484 f_{\text {cyl, inplace }}
$$

Diámetro $5 \mathrm{~cm}$ en condición saturada / Wet 5- cm diameter.

$$
\frac{f_{c, \text { core }(5, \text { wet })}}{f_{c y l, \text { inplace }}}=1.036-0.004474 f_{c y l \text {, inplace }}
$$

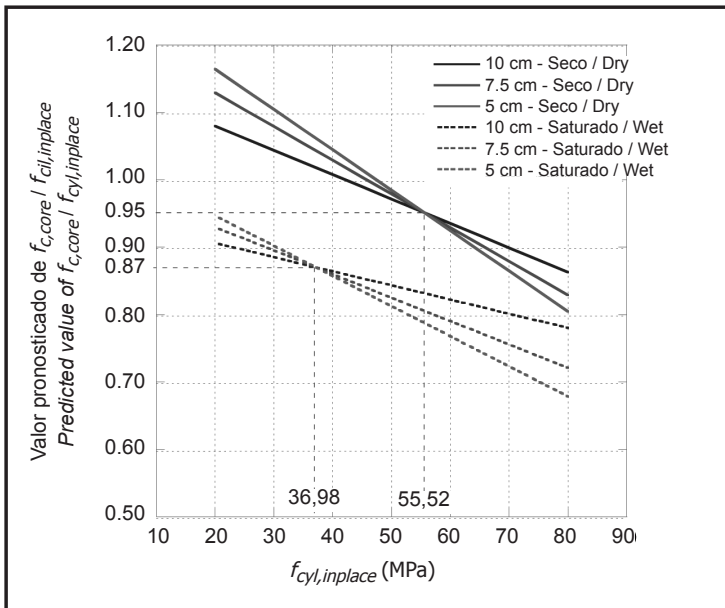

Figura 2. $\tilde{f}_{c, \text { core }} / f_{c y l, \text { inplace }}$ vs $f_{c y l \text {, inplace }}$.

Figure 2. $\tilde{f}_{c, \text { core }} / f_{c y l, \text { inplace }} v s f_{c y l, \text { inplace }}$.

Ahora bien, a partir de las seis rectas de regresión se establece un procedimiento deductivo para la obtención de los factores que corrigen el resultado de la resistencia del testigo, $f_{c, \text { core, }}$ con el objeto de pronosticar la resistencia equivalente in situ, $f_{c y l \text {,inplace. }}$ Es decir, que se pasaría de un modelo aditivo a un modelo multiplicativo.
A deductive procedure based on the regression lines was applied to obtain the correction factors to be applied to core strength, $f_{c, \text { core }}$ to predict the equivalent in-place strength $f_{c y l \text {,inplace }}$. This procedure entailed replacing the above additive model with a multiplicative model. 
Tal como se ha indicado en apartados anteriores, este estudio ha adoptado el modelo multiplicativo de superposición de efectos con valores experimentales obtenidos de un HAC. Dicho modelo es el siguiente [11]:
As noted earlier, in this study the experimental findings for a SCC were applied to a multiplicative approach that assumes superposition of effects, expressed as follows [11]:

$$
\tilde{f}_{\text {cyl,inplace }}=f_{c, \text { core }} F_{\phi} F_{\text {MOIST }} F_{\text {DIR }} F_{\text {BASIC }}
$$

donde:

$f_{c y l \text {,inplace: }}$ Resistencia equivalente in situ pronosticada.

$f_{c, \text { core: }} \quad$ Resistencia a compresión del testigo.

$F_{\phi}$ : $\quad$ Factor de corrección para el diámetro del testigo.

$F_{\text {MOIST }}: \quad$ Factor de corrección para la condición de humedad del testigo.

$F_{D I R}: \quad$ Factor de corrección para la dirección de extracción. $F_{D I R}=1,0$.

$F_{\text {BASIC }}$ Factor de corrección básico, debido a particularidades inherentes a las probetas, tales como, microfisuración, efecto pared, condiciones de contorno, etc.

Debido a que la variable dirección, $D I R$, no fue significativa en el pronóstico de la resistencia equivalente in situ, se ha asumido que el valor para el factor de corrección de dicha variables es $F_{D I R}=1,0$.

Para el procedimiento deductivo se ha tenido en consideración lo indicado en la norma europea UNE-EN 13791:2009. Esta norma señala que existe una equivalencia entre la resistencia de un testigo con diámetro nominal de 10 a $15 \mathrm{~cm}$ y esbeltez igual a 2,0 con la resistencia de una probeta cilíndrica moldeada fabricada y curada bajo las mismas condiciones. Por esta razón se ha elegido el testigo de $10 \times 20 \mathrm{~cm}$ en condición seca como el "testigo de referencia". Igualmente, la norma ACI-214.4R-10 establece este tipo de testigo como referencia para el cálculo de sus factores. En la Figura 3 se describe el procedimiento utilizado para el cálculo de los factores de corrección en el HAC analizado.

En el proceso deductivo ha sido posible calcular los factores de corrección $F_{\phi}, F_{\text {MOIST }}$ y $F_{\text {BASIC }}$ en función de la resistencia de la probeta testigo. Los resultados se muestran en las Tablas 6 y 7.

Se observa que los tres factores de corrección varían según el nivel resistente del hormigón. Lo cual no coincide con la norma ACI-214.4R-10 que establece valores únicos para cada factor de corrección sin importar el nivel resistente del hormigón. En el caso del factor de corrección por diámetro $F_{\phi}$ dicha norma propone utilizar un valor de 1,06 para probetas testigo de $5 \mathrm{~cm}$ en HVT. Si se compara este valor de 1,06 con los resultados de $F_{\phi}$ para el HAC, sólo coincidiría para un rango resistencias entre $60 \mathrm{MPa}$ y $70 \mathrm{MPa}$. where:

$\begin{array}{ll}f_{c y l, \text { inplace: }} & \text { predicted in-place equivalent strength } \\ f_{C, \text { core: }} & \text { core compressive strength } \\ F_{\phi}: & \text { core diameter correction factor } \\ F_{\text {MIIST }}: & \text { core moisture condition correction factor } \\ F_{\text {DIR }}: & \text { casting direction correction factor: } F_{D I R}=1.0 . \\ F_{\text {BASIC: }} & \begin{array}{l}\text { basic factor to correct for inherent specimen } \\ \text { particularities such as microcracking and the } \\ \text { wall or boundary effects }\end{array}\end{array}$

Since the casting direction, DIR, had no significant effect on the prediction of equivalent in-place strength, the value of its correction factor was assumed to be $F_{D I R}=1.0$.

The deductive procedure followed was as laid down in Spanish and European standard UNE-EN 13791:2009. According to that standard, the strength of a core with a nominal diameter of 10 to $15 \mathrm{~cm}$ and a slenderness ratio of 2 is equivalent to the strength of a cylindrical specimen prepared and cured under the same conditions. A dry-cured $10 \times 20-\mathrm{cm}$ core was consequently chosen as the reference core. Standard ACI-214.4R-10 establishes this type of core as the reference for calculating correction factors. Figure 3 describes the procedure used to calculate the correction factors for the SCC analysed.

That procedure was followed to estimate $F_{\phi}, F_{\text {MOIST }}$ and $F_{B A S I C}$ for each range of core strength. The findings are given in Tables 6 and 7.

The three factors were observed to vary with concrete strength. That finding is not consistent with standard ACI-214.4R-10, which lays down only one value for each correction factor, irrespective of concrete strength. The correction factor proposed by the ACI standard for $5-\mathrm{cm}$ diameter cores, 1.06, concurs with the $F_{\phi}$ found in this study for SCCS in the 60-70-MPa strength range only. 


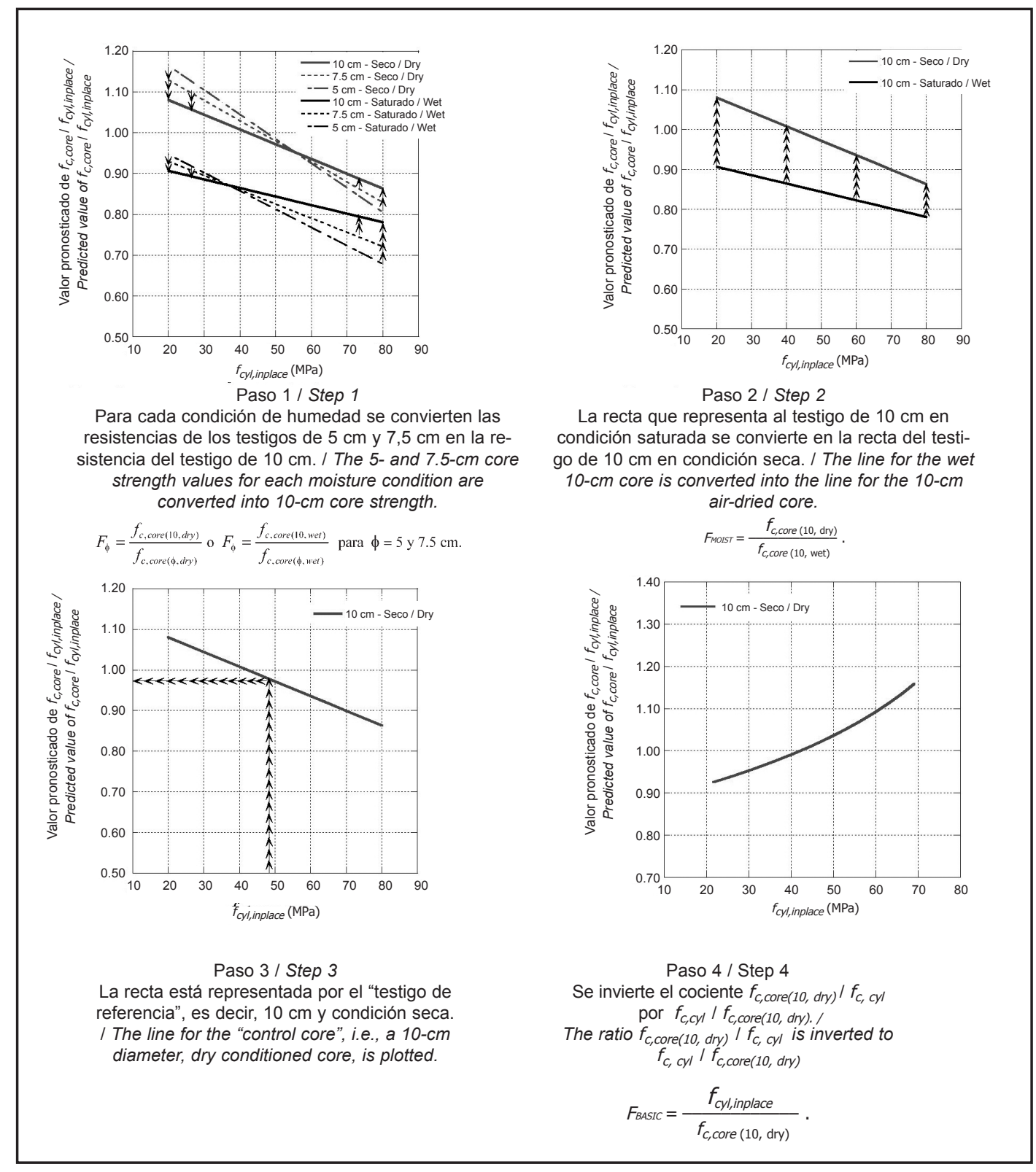

Figura 3. Procedimiento deductivo para el cálculo de los factores de corrección. Figure 3. Deductive procedure for calculating correction factors.

Los resultados del factor de corrección por humedad $F_{\text {MOIST }}$ revelan la gran influencia que presenta el nivel resistente del hormigón en el cociente entre la resistencia de un testigo seco y la de un testigo húmedo. Este efecto podría estar relacionado con las variaciones en la permeabilidad entre los hormigones de resistencias bajas y altas. De igual manera el factor de corrección por humedad $F_{\text {MOIST }}$ propuesto por ACI-214.4R-10 para el HVT al utilizar testigos húmedos es de 1.09 y en el caso del HAC este factor de corrección varía entre 1,12 y 1,19 según el nivel resistente.

Con el objeto de comparar los valores obtenidos del factor de corrección $F_{B A S I C}$ para el HAC con algunos
Concrete strength also proved to affect the difference in dry and moist core strength, and therefore the core moisture correction factor, $F_{\text {MoIs }}$. That effect may be related to the variations in permeability between low- and high-strength concretes. The correction factor for moist cores, $F_{\text {MOIST }}$ proposed in ACI-214.4R-10 is 1.09 for CVC and from 1.12 to 1.19 for SCC, depending on strength.

Figure 4 compares the values obtained here for the $F_{\text {BASIC }}$ correction factor in SCCS to the factors proposed in 
propuestos por diferentes normas e investigadores para el HVT, se ha construido la Figura 4. Recordemos que el factor de corrección $F_{\text {BASIC }}$ se ha obtenido de comparar la resistencia de la probeta cilíndrica de $15 \times 30 \mathrm{~cm}$ y la resistencia de la probeta testigo de $10 \times 20 \mathrm{~cm}$ en condiciones similares de compactación y conservación (ambos secos), es decir, que se estarían evaluando las variables restantes que son difíciles de medir por sí solas, nos referimos a las que son intrínsecas a las probetas (posible microfisuración debido a la extracción, efecto pared, efecto contorno, etc.).

En esta Figura 4 se observan los valores de los factores de corrección $F_{B A S I C}$ obtenidos para el HAC mediante rectas escalonadas que han sido obtenidas después de transformar la línea de segundo grado del paso 4 del procedimiento deductivo. Asimismo, con datos recopilados en la tesis doctoral de Rojas, L. (7) se ha representado la línea de ajuste con los resultados de otras investigaciones que evaluaron la relación $f_{c y l \text {, inplace }} / f_{c \text {, core }}$ en elementos de HVT cuyas especificaciones fueron similares a las del presente estudio. Además, se muestran las dos rectas que representan los valores constantes ofrecidos por la instrucción española EHE-08 y la norma americana ACI 214.4R-10 relacionado con el factor de corrección $F_{\text {BASIC }}$

Es evidente que el comportamiento entre la resistencia equivalente in situ y la resistencia del testigo es diferente para los dos tipos de hormigones. En el caso del HAC estudiado no deben ser utilizados los factores de corrección $F_{B A S I C}$ propuestos para el HVT debido a que se estaría dando un valor mayorado de la resistencia equivalente in situ. standards and by other authors for CVCS. As described earlier, correction factor $F_{B A S I C}$ was obtained by comparing the strength of $15 \times 30-\mathrm{cm}$ cylindrical specimens to the strength of $10 \times 20-\mathrm{cm}$ cores made from similarly consolidated and conditioned (dry in both cases) concrete. In other words, this comparison indirectly assessed the "other" variables (intrinsic features such as possible microcracking due to drilling, or the wall and boundary effects) that are difficult to measure directly.

Figure 4 shows the $F_{\text {BASIC }}$ values observed for SCC as tiered horizontal lines obtained by transforming the second-degree line in step 4 of the deductive procedure. In addition, the figure shows the curve fitted to the data from other

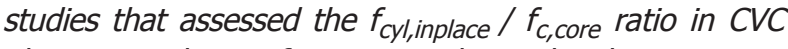
elements with specifications similar to the characteristics of the present materials -drawn from the compilation in Rojas' PhD. thesis (7). The constant values for the $F_{B A S I C}$ factor specified in Spanish code EHE-08 and American standard ACI 214.4R-10 are likewise shown.

The obvious difference in the equivalent in-place strength and core strength ratios between the two types of concrete is a clear indication that the $F_{\text {BASIC }}$ correction factors proposed for CVC should not be used for the SCC studied, for they would overestimate equivalent in-place strength, particularly for lower strengths.

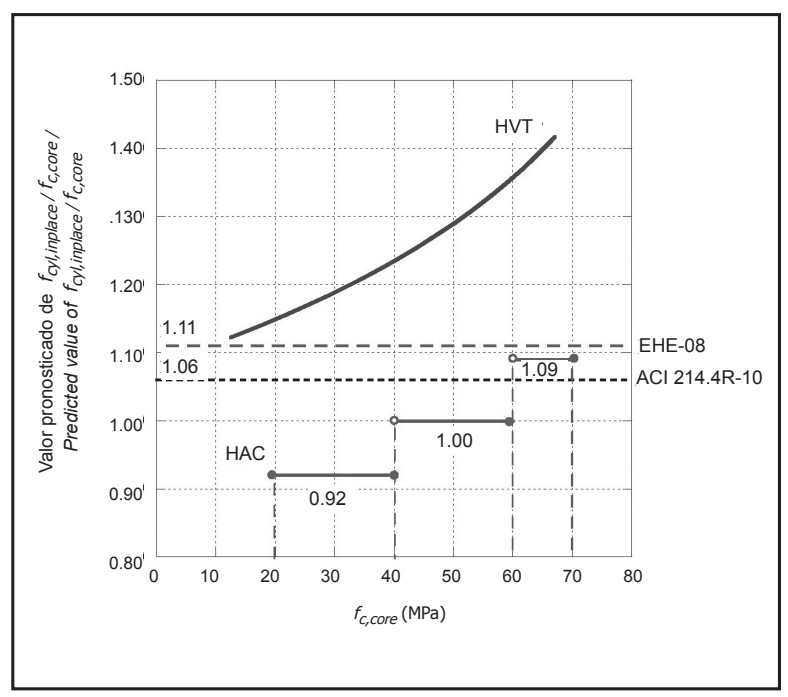

Figura 4. Factor de corrección $F_{\text {BASIC }}$ entre HAC y HVT.

Figure 4. $F_{\text {BASIC }}$ correction factors derived from differences in SCC and CVC values. 
En la Figura 4 también se observa que la línea de ajuste que representa al HVT se encuentra por encima de los valores recomendados por la instrucción EHE-08 y la norma ACI $214.4 \mathrm{R}-10$, es decir, 1,11 y 1,06 respectivamente. En este sentido los valores ofrecidos por estas normas están del lado de la seguridad, aunque en mayor medida para los hormigones de altas resistencias.

\section{Ejemplo práctico}

Se desea estimar la resistencia in situ de un pilar en hormigón autocompactante, el cual no va a estar nunca saturado en condiciones de servicio. Para ello se han extraído dos probetas testigo de $5 \mathrm{~cm}$ de diámetro con una relación longitud/diámetro igual a 2 . Una de las probetas se ha extraído de la parte más alta, y la otra de la más baja. Estos testigos han sido sumergidos en agua durante 48 horas antes de su rotura, por condiciones de norma de ensayo. Los resultados de resistencia a compresión de las probetas fueron 32,8 MPa y 33,2 MPa.

Definición de factores

Con el objeto de convertir la resistencia del testigo de $5 \mathrm{~cm}$ en el testigo de referencia de $10 \mathrm{~cm}$ obtenemos
Figure 4 also shows that the equivalent in-place strength / core strength ratios for CVC are higher than the values recommended in code EHE-08 and standard ACI $214.4 R-10$, i.e., 1.11 and 1.06, respectively. The values set out in these standards are on the safety side, particularly for high-strength concrete.

\section{Practical example}

Two 5-cm cores with a length/diameter ratio of 2 were drilled to estimate the strength of a self-consolidated, inplace concrete column, assuming consistently dry service conditions. One of the cores was drilled in the uppermost and the other in the lower part of the column. The cores were then soaked for 48 hours and subsequently tested to failure. Their compressive strength was found to be 32.8 and $33.2 \mathrm{MPa}$.

\section{Estimating correction factors}

The 5-cm core strength was converted into $10-\mathrm{cm}$ strength by applying the respective core diameter correction

Tabla 6 / Table 6

Propuesta de factores de corrección para el diámetro del testigo, $F_{\phi}$. Proposed core diameter correction factors, $F_{\phi}$.

\begin{tabular}{|c|c|c|c|c|}
\hline & \multicolumn{2}{|c|}{$F_{\phi}$ para testigos secos / for dry cores } & \multicolumn{2}{c|}{$F_{\phi}$ para testigos saturados / for wet cores } \\
\hline $\begin{array}{c}\boldsymbol{f}_{c, \text { core }} \\
(\mathrm{MPa})^{*}\end{array}$ & $\begin{array}{c}\text { Si se extraen testigos de } \mathbf{5} \mathrm{cm} \\
\text { / For 5-cm cores }\end{array}$ & $\begin{array}{c}\text { Si se extraen testigos de } \mathbf{7 . 5} \mathrm{cm} \\
\text { / For 7.5-cm cores }\end{array}$ & $\begin{array}{c}\text { Si se extraen testigos de } \mathbf{5} \mathrm{cm} \\
\text { / For 5-cm cores }\end{array}$ & $\begin{array}{c}\text { Si se extraen testigos de } 7.5 \mathrm{~cm} \\
\text { / For 7.5-cm cores }\end{array}$ \\
\hline $20-30$ & 0.93 & 0.96 & 0.97 & 0.98 \\
\hline $30-40$ & 0.95 & 0.97 & 1.01 & 1.00 \\
\hline $40-50$ & 0.98 & 0.99 & 1.06 & 1.03 \\
\hline $50-60$ & 1.01 & 1.01 & 1.13 & 1.07 \\
\hline $60-70$ & 1.06 & 1.03 & $* *$ & $* *$ \\
\hline
\end{tabular}

* Es la que se obtiene con testigos de $5 \mathrm{~cm}$ y de $7,5 \mathrm{~cm}$. / * Strength obtained with 5 - and 7.5-cm cores.

${ }^{* *}$ Fuera del rango de los resultados experimentales. $/{ }^{* *}$ Outside the range of experimental results.

Tabla 7 / Table 7

Propuesta de factores de corrección para la condición de humedad del testigo, $F_{\text {MOIST }}$ y para las variables intrínsecas, $F_{\text {BASIC }}$. Proposed correction factors for core moisture condition, $F_{\text {MoIST, }}$ and intrinsic variables, $F_{\text {BASIC}}$.

\begin{tabular}{|c|c|}
\hline $\boldsymbol{f}_{\boldsymbol{c , \text { core }}}(\mathbf{M P a})^{*}$ & $\boldsymbol{F}_{\text {MOIST }}$ \\
\hline $20-30$ & 1.19 \\
\hline $30-40$ & 1.17 \\
\hline $40-50$ & 1.14 \\
\hline $50-60$ & 1.12 \\
\hline
\end{tabular}

\begin{tabular}{|c|c|}
\hline $\boldsymbol{f}_{\boldsymbol{C}, \text { core }}(\mathbf{M P a})^{* *}$ & $\boldsymbol{F}_{\text {BASIC }}$ \\
\hline $20-40$ & 0.92 \\
\hline $40-60$ & 1.00 \\
\hline $60-70$ & 1.09 \\
\hline & \\
\hline
\end{tabular}

${ }^{*}$ Resistencia corregida a testigo de $10 \mathrm{~cm}$ y a condición saturada. ${ }^{* *}$ Resistencia corregida a testigo de $10 \mathrm{~cm}$ y a condición seca. * Strength corrected for 10-cm wet core ** Strength corrected for 10-cm dry core. 
de la Tabla 6 en factor de corrección por diámetro, $F_{\phi}$. Para un rango de resistencias entre $30 \mathrm{MPa}$ y $40 \mathrm{MPa}$ y para testigos en condición saturada este factor de corrección es igual a 1,01.

Una vez corregido el diámetro se procede a convertir la resistencia del testigo de estado saturado a estado seco (condición de referencia). De la Tabla 7 para el mismo rango de resistencia (30-40 $\mathrm{MPa}$ ) se obtiene un factor de corrección por humedad, $F_{\text {MOIST }}$, igual a 1,17.

El factor de corrección por dirección, $F_{D I R}$, es igual a 1,00 . Según los resultados de este estudio en el HAC la dirección de extracción no es estadísticamente significativa en el resultado de la resistencia del testigo.

Finalmente el factor $F_{\text {BASIC }}$ que evalúa las restantes variables intrínsecas se obtiene de la Tabla 7. Para un rango de resistencias entre 20-40 MPa este factor es igual a 0,92 .

Es decir, que la resistencia estimada in situ para el pilar en HAC es la siguiente (Tabla 8): factor, $F_{\phi}$. According to Table 6, the factor for wet cores in the 30-40-MPa strength range is 1.01 .

After correcting for the diameter, wet core strength was converted to dry strength (reference condition). The core moisture correction factor, $F_{\text {MOIST, }}$ for the same strength range (30-40 $\mathrm{MPa})$ was found in Table 7 to be 1.17 .

The casting direction factor, $F_{D I R}$, was taken to be equal to 1.00, for, according to the present findings on SCC, casting direction has no statistically significant effect on core strength.

Lastly, the $F_{\text {BASIC }}$ factor for other intrinsic variables was applied. The value found in Table 7 for strength in the 20-40-MPa range was 0.92.

The in-place strength for the SCC column estimated from the aforementioned operations is given in Table 8 below.

Tabla 8 / Table 8

Cálculo de la resistencia estimada in situ para un pilar en HAC. Estimated in-place strength for a SCC column.

\begin{tabular}{|c|c|}
\hline Testigo 1 / Core 1 & Testigo 2 / Core 2 \\
\hline$\tilde{f}_{\text {cyl, inplace }}=32.8 \times 1.01 \times 1.17 \times 1.00 \times 0.92=35.7 \mathrm{MPa}$ & $\tilde{f}_{\text {cll, inplace }}=33.2 \times 1.01 \times 1.17 \times 1.00 \times 0.92=36.6 \mathrm{MPa}$ \\
\hline
\end{tabular}

Resistencia estimada in situ (valor mínimo) / Estimated in-place strength (minimum value) = 35.7 MPa

\subsection{Factores que convierten la resistencia de la probeta cúbica en la resistencia de la probeta cilíndrica}

Mediante el método de mínimos cuadrados ordinarios (9) se ha obtenido un modelo ajustado con una muy buena eficacia predictiva, que permite pronosticar la resistencia en probeta cilíndrica a través de la resistencia en probeta cúbica. Los contrastes realizados a dicho modelo han permitido no rechazar las hipótesis de homoscedasticidad y normalidad de los residuos. Este modelo tiene por ecuación [12]:

\subsection{Factors for converting cubic to cylindrical specimen strength}

A model derived from ordinary minimum squares (9) was able to reliably predict cylindrical specimen strength from cubic specimen strength. Neither the homoscedasticity nor the normality of residuals hypothesis was rejected when the model was contrasted. The respective equation is shown below [12]:

$$
\tilde{f}_{c, c y l}=-2.260+0.9620 f_{c, \text { cub }} .
$$

A partir de la ecuación anterior y teniendo en cuenta la expresión utilizada para la predicción de la resistencia de la probeta cilíndrica a partir de la cúbica [13]:
Based on the above equation and the expression used to predict cylindrical from cubic specimen strength [13];

$$
\tilde{f}_{c, c y l}=f_{c, c u b} F_{c u b},
$$


es posible obtener el factor de corrección, $F_{\text {cub }}$ mediante la expresión [14]: the $F_{c u b}$ correction factor can be obtained from the following expression [14]:

$$
F_{c u b}=\frac{\tilde{f}_{c, c y l}}{f_{c, c u b}}=0.9620-\frac{2.260}{f_{c, c u b}} .
$$

Los factores de corrección $F_{c u b}$ obtenidos a partir de la relación anterior se muestran en la Tabla 9. Los rangos de $f_{c, c u b}$ se han establecido teniendo en cuenta el valor mínimo y máximo obtenido en los resultados experimentales, es decir, 28,6 MPa y 81,7 MPa.

Tal como lo indica la Tabla 9 la tasa de crecimiento de la resistencia en probeta cúbica es prácticamente constante. Además, se presentan diferencias del $1 \%$ en el factor de corrección $F_{c u b}$ entre los rangos de resistencia $f_{c, c u b}$ considerados.

Los resultados obtenidos están en línea con lo especificado por algunas investigaciones realizadas en HVT. Tal es el caso de Mansur \& Islam (10) que obtuvieron una pendiente del ajuste prácticamente igual a 1,0. Estos autores concluyen que la diferencia entre la resistencia en probeta cúbica y probeta cilíndrica es prácticamente una constante independientemente del nivel resistente del hormigón.

En relación con la instrucción EHE-08, los valores propuestos de $F_{c u b}$ para el rango de resistencias ensayado varía entre 0,90 y 0,95. Estos valores son similares a los obtenidos en este estudio, entre 0,90 y 0,93.

También a efectos de comparación, se han tomado como referencia los valores de resistencia característica señalados por el Código Modelo (11) para HVT, los cuales son mostrados en la Tabla 10.

Si ahora se toma como referencia las mismas resistencias características en probeta cilíndrica de la Tabla 10 y se calculan a partir de ellas las resistencias características en probeta cúbica para los HAC, mediante la [12], se puede construir la Tabla 11. En esta tabla se observa que el rango de resistencias en probeta cilíndrica obtenido de la experimentación está delimitado por líneas discontinuas.
The $F_{\text {cub }}$ correction factors obtained from the preceding equation are given in Table 9. The strength ranges listed were delimited by the experimental minimum and maximum $f_{c, \text { cub }}$ values, i.e., 28.6 and 81.7 MPa.

As Table 9 shows, strength rose at a practically constant rate in cubic specimens, while the $F_{c u b}$ values were observed to rise by $1 \%$ from one $f_{c, c u b}$ strength range to the next.

These findings were in keeping with the reports of other authors working with CVC. Mansur and Islam (10), for instance, who obtained a curve with a slope nearly equal to 1.0, concluded that the difference between cubic and cylindrical specimen strength was practically constant irrespective of concrete strength.

Code EHE-08 proposes $F_{\text {cub }}$ values of 0.90 to 0.95 for the strength range tested. The values found here were very similar, from 0.90 to 0.93 .

The characteristic CVC strength values laid down in the 2010 Model Code (11) are given in Table 10, likewise by way of comparison.

Table 11 was constructed by calculating the characteristic cubic specimen strength values by entering the characteristic cylindrical specimen values given in Table 10 in [12]. The range of cylindrical specimen strength values obtained empirically is delimited by dotted lines in the table.

Tabla 9 / Table 9

Factor de corrección $F_{c u b}$.

$F_{c u b}$ correction factors.

\begin{tabular}{|c|c|}
\hline $\boldsymbol{f}_{\boldsymbol{c , \text { cub }}}$ (MPa) & $\boldsymbol{F}_{\text {cub }}$ \\
\hline $30-40$ & 0.90 \\
\hline $40-50$ & 0.91 \\
\hline $50-60$ & 0.92 \\
\hline $60-70$ & 0.93 \\
\hline $70-80$ & 0.93 \\
\hline
\end{tabular}


Tabla 10 / Table 10

Valores de resistencias características de un HVT según el Código Modelo (11). Characteristic CVC strength values as per the 2010 Model Code (11).

\begin{tabular}{|c|c|c|c|c|c|c|c|c|c|c|c|c|}
\hline & $\mathbf{C 1 2}$ & $\mathbf{C 2 0}$ & $\mathbf{C 3 0}$ & $\mathbf{C 4 0}$ & $\mathbf{C 5 0}$ & $\mathbf{C 6 0}$ & $\mathbf{C 7 0}$ & $\mathbf{C 8 0}$ & $\mathbf{C 9 0}$ & $\mathbf{C 1 0 0}$ & $\mathbf{C 1 1 0}$ & $\mathbf{C 1 2 0}$ \\
\hline Cilíndrica/ Cylindrical & 12 & 20 & 30 & 40 & 50 & 60 & 70 & 80 & 90 & 100 & 110 & 120 \\
\hline Cúbica / Cubic & 15 & 25 & 37 & 50 & 60 & 75 & 85 & 95 & 105 & 115 & 130 & 140 \\
\hline
\end{tabular}

Tabla 11 / Table 11

Valores de resistencias características de un HAC. Characteristic SCC strength values.

\begin{tabular}{|c|c|c|c|c|c|c|c|c|c|c|c|c|}
\hline & C12 & C20 & C30 & C40 & C50 & C60 & C70 & C80 & C90 & C100 & C110 & C120 \\
\hline Cilíndrica / Cylindrical & - & - & 30 & 40 & 50 & 60 & 70 & 80 & - & - & - & - \\
\hline Cúbica / Cubic & - & - & 35 & 45 & 55 & 65 & 75 & 85 & - & - & - & - \\
\hline
\end{tabular}

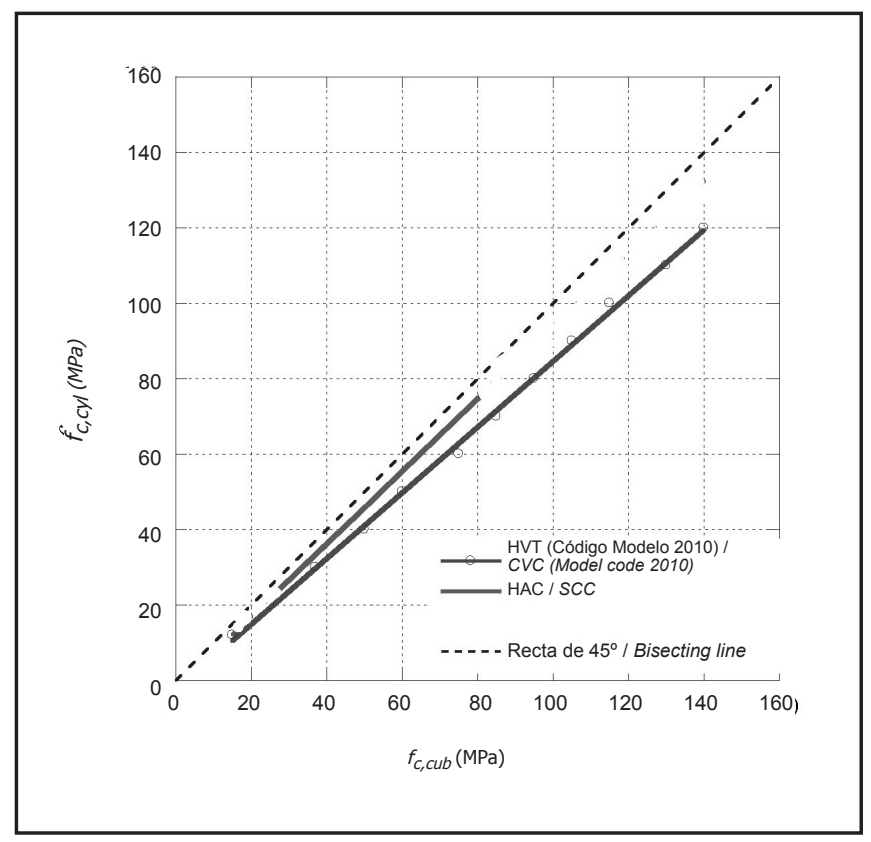

Figura 5. Gráfico $F_{c, c y l}$ vs $F_{c, c u b}$ en HVT y HAC.

Figure 5. Figura 5. $F_{c, c y l}$ vs $F_{c, c u b}$ in $\mathrm{CVC}$ and SCC.

En la Figura 5 se representa tanto la línea de ajuste obtenida para el HAC analizado como la línea de ajuste que aproxima los valores dados por el Código Modelo (11) para HVT. Además el gráfico es complementado con la recta de referencia de $45^{\circ}$. En esta figura se observa claramente que las rectas de ajuste del HVT y del HAC tienden a pasar muy cerca del origen. Es decir, que la tasa de crecimiento de $F_{c, c u b}$ con respecto a $f_{c, c y l}$ es prácticamente constante. Por otro lado, las resistencias características para las probetas cilíndricas y cúbicas se aproximan más en el HAC que en el HVT. En el HAC por
Figure 5 plots the cylindrical compressive strength against the cubic compressive strength of the SCC analysed that resembled the Model Code (11) values for CVC most closely. The $45^{\circ}$ reference line is also represented. The figure shows that the CVC and SCC regression lines tend to pass very close to the origin. In other words, $F_{c, c u b}$ rose in practically constant proportion to $f_{c, c y l}$. Moreover, the characteristics strenght for cylindrical and cubic specimens are closer in SCC than in CVC. Due to self-consolidation, the paste in SCC is more uniform. Consequently, it is possible that 
condiciones de autocompactabilidad los contenidos de pasta son más uniformes y por lo tanto es posible que las diferencias en rigidez entre ambos tipos de probetas sean menos acusadas con el nivel resistente del hormigón.

\section{CONCLUSIONES}

- Se han determinado algunos factores que corrigen el resultado de la resistencia del testigo para la predicción de la resistencia in situ de un HAC. Estos factores de corrección están relacionados con el diámetro del testigo, la dirección de extracción, la condición de humedad del testigo y el conjunto de variables intrínsecas, tales como, microfisuración, efecto pared, condiciones de contorno, etc. Los valores obtenidos para estos factores dependen del nivel resistente del hormigón.

- Se han encontrado diferencias en el cociente entre la resistencia equivalente in situ y la resistencia del testigo para los dos tipos de hormigones (HAC y HVT). Este cociente obtenido para el HAC, no es constante sino que varía con el nivel resistente del hormigón. En este sentido, el valor 1,11 descrito en la instrucción EHE-08 y el valor 1,06 en la norma ACI 214.4R para los HVT no deberían ser utilizados en el HAC de este estudio, debido a que se estaría sobrevalorando el pronóstico de la resistencia equivalente in situ.

- Las diferencias entre los dos tipos de hormigones podrían ser atribuidas fundamentalmente al grado de compactación del hormigón. Es decir, que al realizar correlaciones entre las probetas testigos y las moldeadas cilíndricas la forma de compactación es más parecida en las probetas de HAC que en las de HVC.

- Para el HAC de este estudio se han obtenido factores que convierten la resistencia de la probeta cúbica en la resistencia de la probeta cilíndrica. Es decir, que para resistencias en probeta cúbica entre $30 \mathrm{MPa}$ y $80 \mathrm{MPa}$ estos factores de corrección varían de 0,90 a 0,93. Dichos factores presentan una tasa de crecimiento prácticamente constante, y desde luego muy pequeña, para el rango de resistencias investigado. Además, tienen similitud con los valores propuestos por la instrucción EHE-08 pero difieren de los obtenidos del Código Modelo (2010).

\section{AGRADECIMIENTOS}

Al Laboratorio Central del Instituto Técnico de Materiales y Construcciones (INTEMAC), al Instituto Español del Cemento y sus Aplicaciones (IECA) y a la empresa de prefabricados PRAINSA un agradecimiento especial por el soporte económico y el acompañamiento durante la experimentación. the differences in stiffness between the two types of specimens are less pronounced with the variation of the strength of concrete.

\section{CONCLUSIONS}

- Several correction factors were determined for predicting in-place SCC strength from core strength, namely for core diameter, casting direction, core moisture and a series of jointly considered intrinsic variables such as microcracking and the wall and boundary effects. The values obtained for these factors were observed to depend on concrete strength.

- The two types of concrete studied (SCC and CVC) exhibited differences in the equivalent in-place strength and core strength ratio. In SCC that ratio is not constant, but varies with concrete strength. The values laid down in Spanish code EHE-08 and ACI 214.4R for CVC (1.11 and 1.06, respectively) are not applicable to the SCC studied here, for they would overestimate the equivalent in-place strength.

- The differences between the two types of concretes may be essentially attributed to the degree of consolidation. Namely when performing correlations between molded cylinders and cores, compaction is more similar in samples of SCC that CVC.

- Factors for converting cubic to cylindrical specimen strength in SCC were also calculated in this study. These factors varied scantly for cubic specimens in the strength range studied (30 to $80 \mathrm{MPa}$ ). The factors found 0.90 to 0.93 , were similar to the EHE-08 proposals but differed from the 2010 Model Code values.

\section{ACKNOWLEDGMENTS}

Thanks go to the Instituto Técnico de Materiales y Construcciones (INTEMAC) Central Laboratory and the Instituto Español del Cemento y sus Aplicaciones (IECA); in particular, the financial and material support received from PRAINSA is gratefully acknowledged. 


\section{BIBLIOGRAFÍA / BIBLIOGRAPHY}

(1) American Concrete Institute. ACI 214.4R. Guide for obtaining cores and interpreting compressive strength results, 2010.

(2) Comisión Permanente Hormigón. EHE-08: Instrucción de hormigón estructural. Ministerio de Fomento. Madrid, 2008.

(3) Pérez, M.: "Relación entre la resistencia a compresión de probetas testigo y probetas normalizadas", Mater .Construcc., 48 (249), 45-53 (1998). http://dx.doi.org/10.3989/mc.1998.v48.i249.486

(4) Yi, S.T.; Yang, E. I.; Choi, J. C.: "Effect of specimen sizes, specimen shapes, and placement directions on compressive strength of concrete", Nuclear Engineering and Design, 2006. 236(2), 115-127. http://dx.doi.org/10.1016/j.nucengdes.2005.08.004

(5) Domone, P. L.: "A review of the hardened mechanical properties of self-compacting concrete". Cement \& Concr. Comp. 29, 2007, 1-12. http://dx.doi.org/10.1016/j.cemconcomp.2006.07.010

(6) AENOR. UNE-EN 12390-3: Ensayos de hormigón endurecido. Parte 3: Determinación de la resistencia a compresión de probetas. Madrid, 2009.

(7) Rojas, L.: "Ensayos de información y extracción de probetas testigo en hormigones autocompactantes". Tesis doctoral. E.T.S.I de Caminos, Canales y Puertos. Universidad Politécnica de Madrid, 2012.

(8) Rojas, L.; Fernández, J.; López, J.C.: "Rebound Hammer, Pulse Velocity, and Core Tests in Self-Consolidating Concrete", ACI Materials Journal 109 (2) (Mar/Apr 2012), 235-243.

(9) ACHE, Asociación Científico-Técnica del Hormigón Estructural. Grupo de trabajo 2/4. Monografía M-19: Modelos lineales aplicados al hormigón estructural. Madrid, 2012.

(10) Mansur, M. A.; Islam, M. M.: Interpretation of concrete strength for nonstandard specimens. Journal of Materials in Civil Engineering, 2002. 14(151).

(11) CEB-FIP, Model Code: Comite Euro-International du Béton. Bulletin d'Information No 213/214. Ed. Thomas Telford, London, 2010. 